Bull. Korean Math. Soc. 51 (2014), No. 4, pp. 923-931

http://dx.doi.org/10.4134/BKMS.2014.51.4.923

\title{
AUTOCOMMUTATORS AND AUTO-BELL GROUPS
}

\author{
Mohammad Reza R. Moghaddam, Hesam Safa, and Azam K. Mousavi
}

\begin{abstract}
Let $x$ be an element of a group $G$ and $\alpha$ be an automorphism of $G$. Then for a positive integer $n$, the autocommutator $[x, n \alpha]$ is defined inductively by $[x, \alpha]=x^{-1} x^{\alpha}=x^{-1} \alpha(x)$ and $\left[x, n+1_{n+1} \alpha\right]=\left[\left[x,{ }_{n} \alpha\right], \alpha\right]$. We call the group $G$ to be $n$-auto-Engel if $\left[x,_{n} \alpha\right]=[\alpha, n x]=1$ for all $x \in G$ and every $\alpha \in \operatorname{Aut}(G)$, where $[\alpha, x]=[x, \alpha]^{-1}$. Also, for any integer $n \neq 0,1$, a group $G$ is called an $n$-auto-Bell group when $\left[x^{n}, \alpha\right]=\left[x, \alpha^{n}\right]$ for every $x \in G$ and each $\alpha \in \operatorname{Aut}(G)$. In this paper, we investigate the properties of such groups and show that if $G$ is an $n$-auto-Bell group, then the factor group $G / L_{3}(G)$ has finite exponent dividing $2 n(n-1)$, where $L_{3}(G)$ is the third term of the upper autocentral series of $G$. Also, we give some examples and results about $n$-auto-Bell abelian groups.
\end{abstract}

\section{Introduction}

Let $G$ be a group and let $\operatorname{Aut}(G)$ denote the automorphism group of $G$. For $\alpha \in \operatorname{Aut}(G)$ and $x \in G$, the autocommutator of $x$ and $\alpha$ is defined to be $[x, \alpha]=x^{-1} x^{\alpha}=x^{-1} \alpha(x)$. The absolute centre and the autocommutator subgroup of $G$ are the subgroups $L(G)=\{x \in G:[x, \alpha]=1$ for all $\alpha \in \operatorname{Aut}(G)\}$ and $K(G)=\langle[x, \alpha]: x \in G, \alpha \in \operatorname{Aut}(G)\rangle$, respectively (see [6]). Clearly, the absolute centre is a characteristic subgroup contained in the centre of $G$ and the autocommutator subgroup is a characteristic subgroup containing the derived subgroup of $G$. Hegarty [6] uses the notation $G^{*}$ for $K(G)$ and proves that if $G / L(G)$ is finite, then so is $K(G)$. Autocommutator subgroup and absolute centre are already studied in $[3,11]$.

Let $n$ be a positive integer. The autocommutator $\left[x,{ }_{n} \alpha\right]$ is defined inductively by $\left[x,_{1} \alpha\right]=[x, \alpha]$ and $\left[x,_{n} \alpha\right]=\left[\left[x_{n-1} \alpha\right], \alpha\right]$ for $n \geq 2$. The group $G$ is said to be $n$-auto-Engel if $\left[x,_{n} \alpha\right]=\left[\alpha,_{n} x\right]=1$ for all $x \in G$ and every $\alpha \in \operatorname{Aut}(G)$, where $[\alpha, x]=[x, \alpha]^{-1}$. Auto-Engel groups are already studied by Moghaddam et al. (see [9]).

Received May 21, 2012; Revised August 16, 2012.

2010 Mathematics Subject Classification. Primary 20D45, 20F12; Secondary 20E36, $20 \mathrm{D} 15$.

Key words and phrases. $n$-auto-Bell group, autocentral series, autocommutator subgroup, $n$-auto-Engel group, $n$-Bell group. 
For any integer $n \neq 0,1$, a group $\mathrm{G}$ is called $n$-auto-Bell if $\left[x^{n}, \alpha\right]=\left[x, \alpha^{n}\right]$ for every $x \in G$ and $\alpha \in \operatorname{Aut}(G)$. In particular, a group $G$ satisfying the previous identity for all inner automorphisms $\alpha \in \operatorname{Inn}(G)$ is an $n$-Bell group. The study of $n$-Bell groups was the subject of several articles, see for instance Brandl and Kappe [1], Kappe and Morse [8], Delizia et al. [4] and Tortora [13].

A group $G$ is called $n$-Kappe if the factor group $G / R_{2}(G)$ has finite exponent dividing $n$, where $R_{2}(G)=\{g \in G:[g, x, x]=1$ for all $x \in G\}$ is the set of all right 2-Engel elements of $G$. It is well known that every $n$-Bell group is $n(n-1)$-Kappe (see Brandl and Kappe [1]).

In [9], it is proved that the set of all right 2-auto-Engel elements of $G$, $A R_{2}(G)=\{g \in G:[g, \alpha, \alpha]=1$ for all $\alpha \in \operatorname{Aut}(G)\}$ is a characteristic subgroup of $G$. Here, we call a group $G$ an $n$-auto-Kappe group when the factor group $G / A R_{2}(G)$ has finite exponent dividing $n$. In this paper, we study some connections of such groups with $n$-auto-Bell groups.

Also, Delizia et al. [4] proved that for an $n$-Bell group $G$, the exponent of $G / Z_{3}(G)$ divides $2 n(n-1)$.

In [10], Moghaddam et al. studied the concept of lower autocentral series and its properties. We define the upper autocentral series by a similar manner. The $n$-th absolute centre of $G$ is defined in the following way: $L_{1}(G)=L(G)$ and $L_{n}(G)=\left\{x \in G:\left[x, \alpha_{1}, \alpha_{2}, \ldots, \alpha_{n}\right]=1\right.$ for all $\left.\alpha_{i} \in \operatorname{Aut}(G)\right\}$. One obtains an ascending chain of characteristic subgroups of $G$ as follows:

$$
1=L_{0}(G) \leq L_{1}(G) \leq \cdots \leq L_{n}(G) \leq \cdots,
$$

which we may call the upper autocentral series of $G$.

In Section 3, we show that if $G$ is an $n$-auto-Bell group, then the factor group $G / L_{3}(G)$ has finite exponent dividing $2 n(n-1)$.

\section{Auto-Bell and auto-Kappe groups}

First, we state a result about 2-auto-Engel groups, which is proved in [9].

Lemma 2.1 ([9]). Let $G$ be a 2-auto-Engel group. Then for every $x, y \in G$, $\alpha \in \operatorname{Aut}(G)$ and $n \in \mathbb{Z}$ the following properties hold:
(a) $\left[x, x^{\alpha}\right]=1$;
(b) $\left[x, \alpha^{n}\right]=[x, \alpha]^{n}=\left[x^{n}, \alpha\right]$
(c) $\left[x^{\alpha}, y\right]=\left[x, y^{\alpha}\right]$;
(d) $[\alpha, x, y]=[\alpha, y, x]^{-1}$.

By the above lemma, every 2-auto-Engel group is an $n$-auto-Bell group for any integer $n \neq 0,1$. Now, suppose that $G$ is a 2-auto-Bell group. Then the identity $\left[x^{2}, \alpha\right]=\left[x, \alpha^{2}\right]$ implies that $\left([x, \alpha]^{x}[x, \alpha]\right)^{\alpha^{-1}}=\left([x, \alpha][x, \alpha]^{\alpha}\right)^{\alpha^{-1}}$. Hence $\left([x, \alpha]^{\alpha^{-1}}\right)^{x}=[x, \alpha]$ and so $\left[x, \alpha, \alpha^{-1} \varphi_{x}\right]=1$, where $\varphi_{x}$ is the inner automorphism defined by $x$. If we replace the automorphism $\alpha$ by $\varphi_{x} \alpha^{-1}$, then we have $\left[\left[x, \alpha^{-1}\right]\left[x, \varphi_{x}\right]^{\alpha^{-1}}, \alpha\right]=1$. Hence $[x, \alpha, \alpha]=1$ and since a right 2auto-Engel element is also a left one (see [9]), $G$ is a 2-auto-Engel group. Thus for any 2-auto-Bell group $G$, we have $[G, \alpha] \subseteq C_{G}(\alpha)$ for every $\alpha \in \operatorname{Aut}(G)$ 
and hence $[\operatorname{Aut}(G), x, x]=1$ for every $x$ in $G$ (i.e., $x$ is also a left 2 -auto-Engel element). Therefore

$$
\operatorname{Aut}(G)=\mathcal{A}(G)=\left\{\alpha \in \operatorname{Aut}(G): x x^{\alpha}=x^{\alpha} x \text { for all } x \in G\right\},
$$

the set of commuting automorphisms of the group $G$ (see [2]). It is easy to see that every 2-auto-Bell group satisfies the identity $\alpha(x) \alpha^{-1}(x)=x^{2}$. In Section 4 , we discuss a family of infinitely many non-abelian finite 2-groups which are 2-auto-Bell.

In what follows, we determine the structure of the abelian 2-auto-Bell groups. Let $G=\left\langle x, y: x^{4}=y^{2}=1, x y=y x\right\rangle \cong \mathbb{Z}_{4} \times \mathbb{Z}_{2}$. Consider the automorphism $\alpha$ of $G$ given by $\alpha(x)=x y$ and $\alpha(y)=y x^{2}$. Clearly, $[x, \alpha, \alpha]=x^{2}$ and hence $G$ is not a 2-auto-Bell group. Now, assume that $G$ is a 2-auto-Bell abelian group, then for the automorphism $\alpha: x \mapsto x^{-1}$, we have $x^{4}=[x, \alpha, \alpha]=1$ for every $x \in G$. Therefore $G$ is a direct sum of cyclic groups of order 2 or 4 . On the other hand, $\left[x, \alpha^{4}\right]=[x, \alpha]^{4}=1$ and so $\exp (\operatorname{Aut}(G))$ divides 4. Using the above example and the fact that $\mathbb{Z}_{2} \times \mathbb{Z}_{2}$ and $\mathbb{Z}_{4} \times \mathbb{Z}_{4}$ have an automorphism of order 3 , it follows that $G \cong 1, \mathbb{Z}_{2}$ or $\mathbb{Z}_{4}$. Recall that the structure of non-abelian 2-auto-Bell (2-auto-Engel) 2-groups is studied in [9].

Now, we discuss the relations between auto-Bell and auto-Kappe groups after some preliminary results.

Lemma 2.2. Let $G$ be an n-auto-Bell group, $x \in G$ and $\alpha \in \operatorname{Aut}(G)$. Then

(i) $\left[x^{n}, \alpha, x^{1-n}\right]=1$;

(ii) $x^{n(1-n)} \in Z\left(x^{\operatorname{Aut}(G)}\right)$, where $x^{\operatorname{Aut}(G)}=\left\langle x^{\alpha}: \alpha \in \operatorname{Aut}(G)\right\rangle$.

Proof. (i) Since $G$ is $n$-auto-Bell,

$$
\left[x^{n}, \alpha\right]^{-x^{-n}}=\left[x^{-n}, \alpha\right]=\left[x^{-1}, \alpha^{n}\right]=\left[x, \alpha^{n}\right]^{-x^{-1}} .
$$

Conjugating with $x$ and taking the inverse yields $\left[x^{n}, \alpha\right]\left[x^{n}, \alpha, x^{1-n}\right]=\left[x, \alpha^{n}\right]$. Hence $\left[x^{n}, \alpha, x^{1-n}\right]=1$.

(ii) Using the Jacobi identity, one obtains $\left[x, \alpha, y^{x}\right]\left[y, x, \alpha^{\varphi_{y}}\right]\left[\alpha, y, x^{\alpha}\right]=1$ for every $x$ and $y$ in $G$ and $\alpha \in \operatorname{Aut}(G)$, where $\varphi_{y}$ is the inner automorphism of $G$ defined by $y$. From this identity and (i) it follows that

$$
1=\left[\alpha, x^{1-n}, x^{n \alpha}\right]=\left[x^{(n-1) \alpha} x^{1-n}, x^{n \alpha}\right]=\left[x^{n-n^{2}}, x^{\alpha}\right] .
$$

Hence $x^{n(1-n)} \in Z\left(x^{\operatorname{Aut}(G)}\right)$.

Proposition 2.3. Every n-auto-Bell group is also $(1-n)$-auto-Bell and hence $n(1-n)$-auto-Bell.

Proof. Since $G$ is an $n$-auto-Bell group, $\left[x^{n}, \alpha^{-1}\right]^{\alpha}=\left[x, \alpha^{-n}\right]^{\alpha}$. Therefore $x^{-n \alpha} x^{n}=x^{-\alpha} x^{\alpha^{1-n}}$ and hence

$$
x^{n-1} x^{(1-n) \alpha} x^{n}=x^{n-1} x^{\alpha^{1-n}} .
$$

So $\left[x^{1-n}, \alpha\right]^{x^{n}}=\left[x, \alpha^{1-n}\right]$. Finally, $\left[x^{1-n}, \alpha\right]\left[x^{1-n}, \alpha, x^{n}\right]=\left[x, \alpha^{1-n}\right]$ and by Lemma 2.2(i), $G$ is a $(1-n)$-auto-Bell group. Clearly, it follows that $G$ is also $n(1-n)$-auto-Bell. 
Observe that an $n$-Bell group need not be a $(-n)$-Bell group, in general. Clearly, by Proposition 2.3 an $n$-auto-Bell group need not be $(-n)$-auto-Bell. In the following theorem, we show that every $n$-auto-Bell group is also $n(n-1)$ auto-Bell.

Theorem 2.4. Every $n$-auto-Bell group is also $n(n-1)$-auto-Kappe and hence $n(n-1)$-auto-Bell.

Proof. Let $G$ be an $n$-auto-Bell group, $x \in G, \alpha \in \operatorname{Aut}(G)$. Using Lemma 2.2(ii) and Proposition 2.3, we get $x^{n(1-n)} \in Z\left(x^{\text {Aut }(G)}\right)$ and hence

$$
1=\left[x^{n(1-n)}, x^{\alpha}\right]=\left[x, x^{n(1-n) \alpha}\right]=\left[x,\left[x^{n(1-n)}, \alpha\right]\right] .
$$

Therefore

$$
\left[\alpha^{n(1-n)}, x, x\right]=1,
$$

and hence, $\alpha^{n(n-1)} \in \mathcal{A}(G)$. So, in every $n$-auto-Bell group, we have the following identity,

$$
\left[x^{n(n-1) \alpha}, x\right]=1=\left[x^{\alpha}, x^{n(n-1)}\right] .
$$

Now, put $m=n(n-1)$. For the $n$-auto-Bell group $G$, it is easy to see that

$$
x^{(n-1) \alpha} x^{\alpha^{1-n}}=x^{n} .
$$

Replacing $x$ by $x^{n}$ yields

$$
x^{n \alpha^{1-n}}=x^{-m \alpha} x^{n^{2}} .
$$

In the equation (4), if we replace $\alpha$ by $\alpha^{-1}$ and conjugate with $\alpha$, we get $x^{n \alpha^{n}}=x^{-m} x^{n^{2} \alpha}$. Now, conjugating the equation (3) with $\alpha^{n}$ and using the latter equality yields

$$
x^{(n-1) \alpha^{n+1}}=x^{n \alpha^{n}} x^{-\alpha}=x^{-m} x^{\left(n^{2}-1\right) \alpha} .
$$

By the equation (2), clearly $\left[x^{m}, \alpha, x\right]=1$ and so $\left[x^{m}, \alpha^{-1}, x\right]^{\alpha}=1$. It follows that

$$
\left[x^{m}, \alpha, x^{\alpha}\right]=1 .
$$

Therefore by Proposition 2.3, equations (4), (5) and (6)

$$
\begin{aligned}
{\left[x^{m}, \alpha, \alpha\right] } & =\left[x^{-m}, \alpha\right]\left[x^{m \alpha}, \alpha\right] \\
& =\left[x^{n}, \alpha^{1-n}\right]\left[x^{(n-1) \alpha}, \alpha^{n}\right] \\
& =x^{-n} x^{n \alpha^{1-n}} x^{(1-n) \alpha} x^{(n-1) \alpha^{n+1}} \\
& =x^{-n} x^{-m \alpha} x^{n^{2}} x^{(1-n) \alpha} x^{n \alpha^{n}} x^{-\alpha} \\
& =x^{m} x^{-m \alpha} x^{(1-n) \alpha} x^{-m} x^{\left(n^{2}-1\right) \alpha} \\
& =x^{\left(-n(n-1)+(1-n)+n^{2}-1\right) \alpha} \\
& =1 .
\end{aligned}
$$


Thus $G$ is $n(n-1)$-auto-Kappe. It also follows that $\left[x^{-1}, \alpha^{-m}\right]^{\alpha}=\left[x^{-1}, \alpha^{-m}\right]$. Therefore $\left[x, \alpha^{m}\right]^{\alpha^{-1}}=\left[x, \alpha^{m}\right]$ and hence by Proposition 2.3 and (1) we get:

$$
\begin{aligned}
{\left[x^{m}, \alpha\right] } & =\left[\left(x^{-m}\right)^{-1}, \alpha\right]=\left[x, \alpha^{-m}\right]^{-x^{-m}} \\
& =\left[x, \alpha^{-m}\right]^{-1}=\left[x, \alpha^{m}\right]^{\alpha^{-m}} \\
& =\left[x, \alpha^{m}\right] .
\end{aligned}
$$

So $G$ is an $n(n-1)$-auto-Bell group.

Remark 2.5. Some connections are held between Kappe and Bell groups, which may not be true for auto-Kappe and auto-Bell groups. For example in [4, Theorem 2.1], it is pointed out that every $n$-Kappe group is an $n^{2}$-Bell group. If $G$ is the elementary abelian 2-group of order 4, then $G$ is a 2-auto-Kappe, but as $G$ has an automorphism of order 3, it cannot be a 4-auto-Bell group.

We end this section by pointing a result, which gives some relations about auto-Bell groups.

Proposition 2.6. Let $G$ be a group and $n \neq 0,1$ be an integer.

(i) If $G$ is an $(n-1)$-auto-Kappe and n-auto-Bell group, then $G$ is also $(n-1)$-auto-Bell.

(ii) If $G$ is an $n$-auto-Kappe and $n$-auto-Bell group, then $G$ is also an $(n+1)$ auto-Bell group.

Proof. (i) Let $x \in G$ and $\alpha \in \operatorname{Aut}(G)$. Since $G$ is an $n$-auto-Bell group (and hence $(1-n)$-auto-Bell) and also an $(n-1)$-auto-Kappe, we get

$$
\left[x^{1-n}, \alpha\right]=\left[x, \alpha^{1-n}\right]=\left[x, \alpha^{n-1}\right]^{-\alpha^{1-n}}=\left[x, \alpha^{n-1}\right]^{-1} .
$$

On the other hand, since $x^{n-1}$ is a right 2-auto-Engel element, it is also a left one and so $\left[\alpha, x^{n-1}, x^{n-1}\right]=1$. Therefore $\left[x^{1-n}, \alpha\right]=\left[x^{n-1}, \alpha\right]^{-1}$. This implies that $\left[x^{n-1}, \alpha\right]=\left[x, \alpha^{n-1}\right]$ and hence $G$ is an $(n-1)$-auto-Bell group.

(ii) Since $G$ is an $n$-auto-Kappe, one may show that $\left[x^{n}, \varphi_{x} \alpha^{-1}, \alpha\right]=1$, where $\varphi_{x}$ is the inner automorphism defined by the element $x$. Replacing $\alpha$ by $\alpha^{-1} \varphi_{x}$ yields $\left[x^{n}, \alpha, \alpha^{-1} \varphi_{x}\right]=1$. Thus $\left[x^{n}, \alpha\right]^{\alpha^{-1}} x=x\left[x^{n}, \alpha\right]$ and hence $\left[x^{n}, \alpha\right] x^{\alpha}=x^{\alpha}\left[x^{n}, \alpha\right]^{\alpha}$. Therefore $x^{-1}\left[x^{n}, \alpha\right] x x^{-1} x^{\alpha}=x^{-1} x^{\alpha}\left[x^{n}, \alpha\right]^{\alpha}$ and from the fact that $G$ is an $n$-auto-Bell group, it follows that $\left[x^{n}, \alpha\right]^{x}[x, \alpha]=$ $[x, \alpha]\left[x, \alpha^{n}\right]^{\alpha}$. This shows that $\left[x^{n+1}, \alpha\right]=\left[x, \alpha^{n+1}\right]$. Thus $G$ is an $(n+1)$ auto-Bell.

\section{Upper autocentral series in auto-Bell groups}

Given a group $G$, the $n$-th autocommutator subgroup of $G$ is

$$
K_{n}(G)=\left\langle\left[x, \alpha_{1}, \alpha_{2}, \ldots, \alpha_{n}\right]: x \in G, \alpha_{1}, \ldots, \alpha_{n} \in \operatorname{Aut}(G)\right\rangle .
$$

It can be easily seen that for every $n \in \mathbb{N}$, the $n$-th autocommutator subgroup is a characteristic subgroup of $G$ containing $\gamma_{n+1}(G)$. Now, we obtain the following series of subgroups

$$
G=K_{0}(G) \geq K(G)=K_{1}(G) \geq K_{2}(G) \geq \cdots \geq K_{n}(G) \geq \cdots,
$$


which is called the lower autocentral series of $G$. In [10], it is proved that for any finite abelian group $G$ and every natural number $n$, there exists a finite abelian group $H$ such that $G \cong K_{n}(H)$.

Now, the $n$-th absolute centre of $G$ is defined inductively by $L_{1}(G)=L(G)$ and $L_{n}(G)=\left\{x \in G:\left[x, \alpha_{1}, \alpha_{2}, \ldots, \alpha_{n}\right]=1\right.$ for all $\left.\alpha_{i} \in \operatorname{Aut}(G)\right\}$. Clearly, the $n$-th absolute centre of $G$ is contained in the $n$-th centre of $G, Z_{n}(G)$. One obtains an ascending chain of characteristic subgroups of $G$ as follows:

$$
1=L_{0}(G) \leq L_{1}(G) \leq \cdots \leq L_{n}(G) \leq \cdots,
$$

which we may call the upper autocentral series of $G$. In the following theorem, we prove that if $G$ is an $n$-auto-Bell group, then $\left[G^{2 n(n-1)}, \alpha, \beta, \gamma\right]=1$ for every $\alpha, \beta, \gamma \in \operatorname{Aut}(G)$.

Theorem 3.1. Let $G$ be an $n$-auto-Bell group. Then the factor group $G / L_{3}(G)$ has finite exponent dividing $2 n(n-1)$.

Proof. First, we show that for any right 2-auto-Engel element $x$ of $G$, the subgroup $x^{\operatorname{Aut}(G)}=\left\langle x^{\alpha}: \alpha \in \operatorname{Aut}(G)\right\rangle$ is abelian. Let $\alpha$ and $\beta$ be automorphisms of $G$. Then

$$
\left[x^{\alpha}, x^{\beta}\right]=\left[x^{\alpha \beta^{-1}}, x\right]^{\beta}=\left[x\left[x, \alpha \beta^{-1}\right], x\right]^{\beta}=\left[\left[x, \alpha \beta^{-1}\right], x\right]^{\beta} .
$$

On the other hand, every right 2-auto-Engel element is also a left 2-auto-Engel element. Hence $\left[\alpha \beta^{-1}, x, x\right]=1$ and this implies that $\left[x^{\alpha}, x^{\beta}\right]=1$ and hence $x^{\text {Aut }(G)}$ is abelian.

Now, by Theorem $2.4, g:=x^{n(n-1)}$ is a right 2-auto-Engel element. So, for each $\alpha \in \operatorname{Aut}(G)$, we have $\left[g, \alpha^{-1}\right]=[g, \alpha]^{-1}$. On the other hand, since $g^{\operatorname{Aut}(G)}$ is abelian, we get $[g, \alpha \beta]=[g, \alpha][g, \beta][g, \alpha, \beta]$ (observe that $[g, \alpha] \in g^{\operatorname{Aut}(G)}$ ) for every $\alpha, \beta \in \operatorname{Aut}(G)$. Hence $[g, \alpha \beta]^{-1}=\left[g, \beta^{-1} \alpha^{-1}\right]$ and the above equality shows that

$$
[g, \alpha, \beta]=[g, \beta, \alpha]^{-1} \text {. }
$$

Now, suppose that $\alpha, \beta$ and $\gamma$ are arbitrary automorphisms of $G$. One may check that the equality $[g, \alpha, \beta \gamma]=[g, \beta \gamma, \alpha]^{-1}$ implies that $[g, \alpha, \beta, \gamma]^{2}=1$ and since $g$ is a 2-auto-Engel element, we obtain $\left[g^{2}, \alpha, \beta, \gamma\right]=1$. Therefore $\left[x^{2 n(n-1)}, \alpha, \beta, \gamma\right]=1$ and this completes the proof.

\section{Abelian $n$-auto-Bell groups}

Clearly, every abelian group is an $n$-Bell group, but this statement is not true for $n$-auto-Bell groups. In what follows, we give some examples of autoBell groups and also discuss some results about $n$-auto-Bell abelian groups. Observe that by Proposition 2.3, in this section we may suppose that $n \geq 2$.

Example 4.1. (i) Let $G$ be a non-periodic abelian group, and consider the inverting automorphism $\alpha \in \operatorname{Aut}(G)$ and a torsion-free element $x \in G$. Then one can easily see that $\left[x^{n}, \alpha\right]=x^{-2 n}$ and $\left[x, \alpha^{n}\right]=x^{(-1)^{n}-1}$. If $G$ is an $n$ auto-Bell group, then we must have $-2 n=(-1)^{n}-1$, and this implies that $n \in\{0,1\}$. So, $G$ cannot be an $n$-auto-Bell group for every integer $n \neq 0,1$. 
(ii) In [7], Jamali constructed the following family of groups. For $m \geq 3$, let $G_{m}$ be a 2-group with the following presentation

$$
\begin{aligned}
G_{m}= & \left\langle a_{1}, \ldots, a_{m}, b: a_{1}^{2}=a_{2}^{4}=\cdots=a_{m}^{4}=1, a_{m-1}^{2}=b^{2},\left[a_{1}, b\right]=1,\right. \\
& {\left.\left[a_{m}, b\right]=a_{1},\left[a_{i-1}, b\right]=a_{i}^{2},\left[a_{j}, a_{k}\right]=1,3 \leq i \leq m, 1 \leq j<k \leq m\right\rangle . }
\end{aligned}
$$

The group $G_{m}$ is of order $2^{2 m}$ with exponent 4 whose automorphism group is isomorphic to $\mathbb{Z}_{2}^{m^{2}}$ and also $Z\left(G_{m}\right) \cong \mathbb{Z}_{2}^{m}$. Clearly, for every $m \geq 3, G_{m}$ is a non-abelian 2-auto-Bell (and hence an $n$-auto-Bell, for every $n \geq 3$ ) group. By using GAP [5], one can check that $G_{3} \cong\left(\mathbb{Z}_{4} \rtimes \mathbb{Z}_{4}\right) \rtimes \mathbb{Z}_{4}$.

(iii) Let $G=\mathbb{Z}_{8} \times \mathbb{Z}_{4} \times \mathbb{Z}_{2} \cong\langle x\rangle \times\langle y\rangle \times\langle z\rangle$. Consider the automorphism $\alpha$ defined by $\alpha(x)=x y, \alpha(y)=x^{2} y z$ and $\alpha(z)=x^{4} y^{2} z$. One can easily check that $1=\left[x^{4}, \alpha\right] \neq\left[x, \alpha^{4}\right]$ and so $G$ is not a 4 -auto-Bell group.

Recall that there are only two non-trivial abelian 2-auto-Bell groups, namely $\mathbb{Z}_{2}$ and $\mathbb{Z}_{4}$

Observe that if $G \cong H \times K$ is an $n$-auto-Bell group, then so are $H$ and $K$. Now, let $G$ be an abelian $n$-auto-Bell group $(n \geq 3)$ and $\alpha$ be the inverting automorphism. Clearly, the identity $\left[x^{n}, \alpha\right]=\left[x, \alpha^{n}\right]$ implies that $\exp (G)$ divides $2 n$ or $2(n-1)$ when $n$ is an even or an odd integer, respectively. By Proposition 2.3, $G$ is also a $(1-n)^{2}$-auto-Bell group. Hence, the exponent of $\operatorname{Aut}(G)$ divides $n(n-2)$ or $(n-1)^{2}$ when $n$ is an even or an odd integer, respectively.

By the above statement, it is easy to see that the 3-auto-Bell abelian groups are actually 2-auto-Bell. Assume that $n=4$. Therefore $G$ is a direct sum of cyclic groups of order 2,4 or 8. Hence, Example 4.1(iii) and the fact that $\mathbb{Z}_{2} \times \mathbb{Z}_{2}, \mathbb{Z}_{4} \times \mathbb{Z}_{4}$ and $\mathbb{Z}_{8} \times \mathbb{Z}_{8}$ have an automorphism of order 3 , show that $G$ is isomorphic to one of the groups $\mathbb{Z}_{2}, \mathbb{Z}_{4}, \mathbb{Z}_{8}, \mathbb{Z}_{2} \times \mathbb{Z}_{4}, \mathbb{Z}_{2} \times \mathbb{Z}_{8}$ or $\mathbb{Z}_{4} \times \mathbb{Z}_{8}$.

Finally, let $G$ be a 5 -auto-Bell abelian group. It is easy to see that $\exp (G)$ and $\exp (\operatorname{Aut}(G))$ divide 8 and 16 , respectively. One may check that the abelian 5 -auto-Bell groups are actually 4-auto-Bell.

Remark 4.2. Let $p$ be an odd prime, $n \geq 6$ and $G$ be an abelian $n$-auto-Bell $p$-group (if any). By the above statement, it is easy to see that $\exp (\operatorname{Aut}(G))$ divides $n$ or $(n-1)$ when $n$ is an even or an odd integer, respectively.

The following theorem may be considered as a criterion for recognition of abelian $p$-groups which are not $n$-auto-Bell.

Theorem 4.3. Let $G$ be a finite abelian $n$-auto-Bell group with $|G|=\prod_{i=1}^{m} p_{i}{ }^{r_{i}}$. Then for every $1 \leq j \leq m$, the numbers $p_{j}\left(p_{j}-1\right)$ and $\prod_{i=1}^{m} p_{i}$ divide $n$ or $(n-1)$ when $n$ is an even or an odd integer, respectively.

Proof. Suppose that an arbitrary prime $p$ divides the order of $G$. Clearly, the Sylow $p$-subgroup $P$ of $G$ is also an $n$-auto-Bell group. If $p=2$ or 3 the result is true. Suppose that $n$ is an even integer and $p \geq 5$. By considering the inverting automorphism, we get $p \mid n$. Let $\alpha: x \mapsto x^{\lambda}$ be an automorphism of $P$, where $1<\lambda<p$ and $(\lambda, p-1)=1$. Then the identity $\left[x^{n}, \alpha\right]=\left[x, \alpha^{n}\right]$ implies that $p \mid\left(\lambda^{n}-n \lambda+n-1\right)$. Therefore $\lambda^{n} \equiv 1(\bmod p)$. 
On the other hand, Euler's theorem implies that $\lambda^{p-1} \equiv 1(\bmod p)$ and since $(\lambda, p-1)=1$, we get $(p-1) \mid n$. Therefore $p(p-1) \mid n$. Similarly, it may be shown that $p(p-1) \mid(n-1)$, if $n$ is an odd integer. Therefore the proof is complete.

The above theorem immediately yields the following corollary.

Corollary 4.4. There is no abelian n-auto-Bell p-group for $n<p(p-1)$.

Proposition 4.5. If $G$ is an abelian $p(p-1) n$-auto-Bell p-group ( $p$ odd and $1 \leq n \leq p-1)$, then $G \cong \mathbb{Z}_{p}$.

Proof. Clearly, $\mathbb{Z}_{p}$ is a $p(p-1) m$-auto-Bell group for every $m \in \mathbb{N}$. It is enough to show that $\mathbb{Z}_{p} \times \mathbb{Z}_{p}$ and $\mathbb{Z}_{p^{k}}(k \geq 2)$ are not $p(p-1) n$-auto-Bell. Since $\operatorname{Aut}\left(\mathbb{Z}_{p} \times \mathbb{Z}_{p}\right) \cong G L(2, p)$ and $\exp (G L(2, p))=p\left(p^{2}-1\right)$, we obtain $1=\left[x^{p(p-1) n}, \alpha\right] \neq\left[x, \alpha^{p(p-1) n}\right]$ for some $x \in \mathbb{Z}_{p} \times \mathbb{Z}_{p}$ and $\alpha \in G L(2, p)$.

Also, since $p^{k}$ does not divide $p(p-1) n$, we get $\left[x^{p(p-1) n}, \alpha\right] \neq\left[x, \alpha^{p(p-1) n}\right]=$ 1 and hence the cyclic group of order $p^{k}(k \geq 2)$ cannot be a $p(p-1) n$-auto-Bell p-group.

Remark 4.6. In the previous proposition, it is not difficult to show that if $n=p$, then $G \cong \mathbb{Z}_{p}$ or $\mathbb{Z}_{p^{2}}$. If $n=p+1$, then $G \cong \mathbb{Z}_{p}$ or $\mathbb{Z}_{p} \times \mathbb{Z}_{p}$ and finally if $n=p+2$, then $G \cong \mathbb{Z}_{p}$. Observe that if $n>p+2$, then the structure of $G$ may depend on the odd prime $p$.

\section{References}

[1] R. Brandl and L.-C. Kappe, On n-Bell groups, Comm. Algebra 17 (1989), no. 4, 787807.

[2] M. Deaconescu, G. Silberberg, and G. L. Walls, On commuting automorphisms of groups, Arch. Math. (Basel) 79 (2002), no. 6, 423-429.

[3] M. Deaconescu and G. L. Walls, Cyclic groups as autocommutator groups, Comm. Algebra 35 (2007), no. 1, 215-219.

[4] C. Delizia, M. R. R. Moghaddam, and A. Rhemtulla, The structure of Bell groups, J. Group Theory 9 (2006), no. 1, 117-125.

[5] The GAP Group, GAP-Groups, Algorithms and Programming, Version 4.4.12, (2008), (http://www.gap-system.org/).

[6] P. V. Hegarty, The absolute centre of a group, J. Algebra 169 (1994), no. 3, 929-935.

[7] A. R. Jamali, Some new non-abelian 2-groups with abelian automorphism groups, J. Group Theory 5 (2002), no. 1, 53-57.

[8] L.-C. Kappe and R. F. Morse, Groups with 3-abelian normal closures, Arch. Math. (Basel) 51 (1988), no. 2, 104-110.

[9] M. R. R. Moghaddam, M. Farrokhi, and H. Safa, Some properties of 2-auto-Engel groups, Submitted.

[10] M. R. R. Moghaddam, F. Parvaneh, and M. Naghshineh, The lower autocentral series of abelian groups, Bull. Korean Math. Soc. 48 (2011), no. 1, 79-83.

[11] M. R. R. Moghaddam and H. Safa, Some properties of autocentral automorphisms of a group, Ricerche Mat. 59 (2010), no. 2, 257-264.

[12] D. J. S. Robinson, Finiteness Conditions and Generalized Soluble Groups, Parts 1 and 2, Springer-Verlag, 1972.

[13] A. Tortora, Some properties of Bell groups, Comm. Algebra 37 (2009), no. 2, 431-438. 
Mohammad Reza R. Moghaddam

Department of Mathematics

Khayyam Higher Education Institute

MASHHAD, IRAN

AND

Centre of Excellence in Analysis on Algebraic Structures

FERdowsi University of MAshHad

IRAN

E-mail address: mrrm5@yahoo.ca

Hesam SAFA

Department of MATHEMATics

Faculty of Basic Sciences

UNIVERSITY OF BOJNORD

BOJNORD, IRAN

E-mail address: hesam.safa@gmail.com

Azam K. Mousavi

Faculty of Mathematical Sciences

INTERNATIONAL BRANCH

FERDOWSi UNIVERSity OF MASHHAD

IRAN

AND

Centre of Excellence in Analysis on Algebraic Structures Ferdowsi University of MashHad

IRAN

E-mail address: akafimoosavi@yahoo.com 